\title{
Analysis of Instability of a Cylindrical Soap Film of Finite Dimensions ${ }^{\dagger}$
}

\author{
S. C. Xu, ${ }^{* \neq} \neq$ L. W. Chang, ${ }^{\S}$ and Lan Xu" \\ Institute of Mechanics, Chinese Academy of Sciences, Beijing, 100080, \\ People's Republic of China, Institute of Chemistry, Chinese Academy of Sciences, \\ Beijing, 100080, People's Republic of China, and College of Chemistry, Peking University, \\ Bejing, 100871, People's Republic of China
}

Received J une 5, 1997. In Final Form: October 30, 1997

\begin{abstract}
By means of experiments of instability of a uniform cylindrical soap film, Boys had showed that the bubble molded by the film is unstable when its length is greater than its circumference. Recently that is generally called the Rayleigh Criterion. In this paper, a linear theory in hydrodynamics is applied to analyze the stability of the cylindrical soap film supported by two equal size disks; all conditions of the stationary wave on the end plates of two disks are given. From here we get that the Rayleigh Criterion on the stability of the cylindrical soap film is proved.
\end{abstract}

\section{Introduction}

C. V. Boys, in his elegant little monograph, ${ }^{1}$ discusses an important phenomenon of the cylindrical soap bubble, which is unstable while its length is larger than its circumference. This experimental fact is generally explained by analogy to the Rayleigh Criterion on the instability of infinite cylindrical liquid columns. ${ }^{2}$ They haveessentially differences; for infinitecolumns, all waves of any wavel ength can propagate, but thestationary waves satisfying the boundary conditions on the end plates of two disks can only exist for the cylindrical bubble.

In the past two decades, the stability of liquid bridges has been extensively investigated in fluid science and materials science in space. ${ }^{3-6}$ Most of the works in ref 3 are based on minimum volume conditions. Bauer ${ }^{5}$ investigated the coupled oscillations of a solidly rotating liquid bridge, but using the boundary condition that the edge contact is free to move. From here it can be determined that the liquid bridge is unstable while its length is larger than half of its circumference. The same conclusion was also obtained in ref 6 . Therefore, the boundary conditions at theends of thefluid are of decisive importance.

In this paper, a linear stability analysis is applied to a cylindrical soap bubble supported by two equal in size disks with contact conditions of the soap film adhering to the edges of theend plates of thetwo disks. Furthermore,

\footnotetext{
* To whom correspondence should be addressed.

† This work was supported by the National Science F oundation of China.

¥ Institute of Mechanics, Chinese Academy of Sciences.

$\S$ Institute of Chemistry, Chinese Academy of Sciences.

"College of Chemistry, Peking University. Present address: Mailbox 92, Department of Chemistry, University of Pennsylvania, Philadel phia, PA 19104-6323.

(1) Boys, C. V. Soap Bubbles and theforces that Mould Them; Society for the Promotion of Christian Knowledge: London, 1890; reprinted by Heinemann: London, 1960.

(2) Adamson, A. W. Physical Chemistry of Surfaces, 5th ed.; Wiley: New York, 1990.

(3) Martinez, I.; Haynes, J. M.; Langbein, D. In Fluid Sciences and Materials Sciencein Space; Walker, H. C., Ed.; Springer Verlag: Berlin, 1987; pp 53-80.

(4) Xu, S. C. Microgravity Sci. Technol. 1993, VI/ 3, 176-183.

(5) Baur, H. F. Acta Astronautica 1982, 9, 547.

(6) Martinez, I. ESA Special Pub. No. 114, 1976.
}

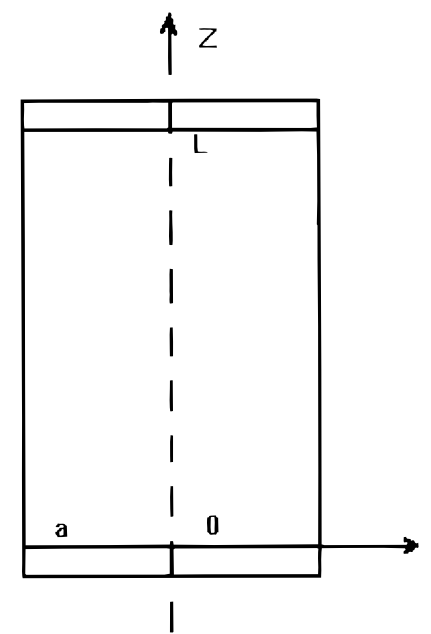

Figure 1. Geometry of the system.

by using the normal mode method, we prove that the sufficient and necessary conditions of stability of a cylindrical soap bubble are that it is unstable while its length is larger than its circumference. This conclusion is well consistent with experiments.

\section{Assumptions and Equilibrium State}

Consider a uniform cylindrical bubble of length $L$ between two disks with radius a (Figure 1 ). At equilibrium, the soap film is essentially a uniform cylinder with radius a.

AccordingtotheYoungand Laplaceequation, thebubble at equilibrium satisfies

$$
\mathrm{p}_{1}-\mathrm{p}_{0}=\frac{\sigma}{\mathrm{a}}
$$

where $\sigma$ is thetension of thesoap film, $\mathrm{p}_{1}$ is thegas pressure in the bubble, and $p_{0}$ is the air pressure out of the bubble. A linear stability method is used to deal with thestability problem of the bubble at equilibrium. Due to a small disturbance, the velocity is much less than the vel ocity of 
sound; the fluid can be considered as an incompressible ideal fluid, taking into account the effect of the tension of the soap film and neglecting the gravitational effect. We assume that the air pressure $p_{0}$ out of bubble is constant during the small disturbance.

\section{Small-Disturbance Hydrodynamic Equations}

The natural coordinates for the system are the cylindrical coordinates $(r, \theta, z)$, the $z$-axis being the axis of the two disks. $z=0$ is the end plate of the lower disk; $z=L$ is the end plate of the upper one. The bubble is a fluid cylinder with radius $a$ and length $L$ at equilibrium.

Assuming the fluid to be only slightly disturbed, the displacement vector of the film disturbed can be written

$$
\mathbf{r}=[a+\zeta(a, \theta, z, t)] \mathbf{e}_{r}+z \mathbf{e}_{r}
$$

The linearized momentum equation is

$$
\rho \frac{\partial \mathbf{u}}{\partial \mathrm{t}}=-\nabla \mathrm{p}
$$

where $\rho$ is the density of the fluid, $\mathbf{u}$ is the disturbed vel ocity of the fluid, and $p$ is the disturbed pressure.

The continuity equation for the incompressible fluid is

$$
\nabla \cdot \mathbf{u}=0
$$

The boundary condition on the disturbed film satisfies

$$
\mathrm{p}-\mathrm{p}_{0}=\sigma \nabla \cdot \mathbf{n} \quad(\mathrm{r}=\mathrm{a}+\xi)
$$

where $\mathrm{p}=\mathrm{p}^{\prime}+\mathrm{p}_{1}$, and $\mathbf{n}$ is theoutward normal unit vector on the film. Its linearization form is

$$
\mathbf{n}=\frac{\frac{\partial \mathbf{r}}{\partial \theta} \times \frac{\partial \mathbf{r}}{\partial z}}{\left|\frac{\partial \mathbf{r}}{\partial \theta} \times \frac{\partial \mathbf{r}}{\partial z}\right|}=\left(1,-\frac{1}{a} \frac{\partial \zeta}{\partial \theta^{\prime}}-\frac{\partial \zeta}{\partial z}\right)
$$

The kinematics boundary condition on the film is

$$
u_{r}=\frac{\partial \zeta}{\partial t} \quad(r=a)
$$

Substituting eq 6 into eq 5, the linearized dynamic boundary condition on the film can be written as

$$
p=-\sigma\left(\frac{\zeta}{a^{2}}+\frac{1}{a^{2}} \frac{\partial^{2} \zeta}{\partial \theta^{2}}+\frac{\partial^{2} \zeta}{\partial z^{2}}\right) \quad(r=a)
$$

At the two end plates $z=0$ and $z=L$ satisfy

$$
\mathrm{u}_{\mathrm{z}}=0
$$

and the contact conditions of the soap film adhering tothe edges of the two disks.

Supposethat the disturbing displacement vector of the fluid is denoted by $\xi(\mathbf{r}, \mathbf{t})$, then by substituting the disturbing vel ocity of the fluid $\mathbf{u}=\partial \xi / \partial t$ into eqs $3-9$, the small-disturbance hydrodynamics equations become

$$
\begin{gathered}
\rho \frac{\partial^{2} \xi}{\partial \mathrm{t}^{2}}=-\nabla \mathrm{p} \\
\nabla \cdot \frac{\partial \xi}{\partial \mathrm{t}}=0 \\
\mathrm{p}+\sigma\left(\frac{\xi \mathrm{r}}{\mathrm{a}^{2}}+\frac{1}{\mathrm{a}^{2}} \frac{\partial^{2} \xi \mathrm{r}}{\partial \theta^{2}}+\frac{\partial^{2} \xi \mathrm{r}}{\partial \mathrm{z}^{2}}\right)=0 \quad(\mathrm{r}=\mathrm{a}) \\
\frac{\partial \xi_{\mathrm{z}}}{\partial \mathrm{t}}(\mathrm{r}, \theta, \mathrm{z}, \mathrm{t})=0 \quad(\mathrm{z}=0, \mathrm{~L})
\end{gathered}
$$

and the contact conditions of the film.

\section{Solutions of Small-Disturbance Hydrodynamics Equations}

Equations 10-13 are a system of initial and boundary value partial differential equations. Equations 10 and 11 yield

$$
\nabla^{2} \mathrm{p}^{\prime}=-\rho \frac{\partial}{\partial \mathrm{t}}\left(\nabla \cdot \frac{\partial \xi}{\partial \mathrm{t}}\right)=0
$$

At the cylindrical coordinate $(r, \theta, z)$, eq 14 takes the form

$$
\frac{1}{r} \frac{\partial}{\partial r}\left(r \frac{\partial p^{\prime}}{\partial r}\right)+\frac{1}{r^{2}} \frac{\partial^{2} p^{\prime}}{\partial \theta^{2}}+\frac{\partial^{2} p^{\prime}}{\partial z^{2}}=0
$$

If the method of separation of variables is employed, the disturbing pressure $p$ is found to be solved in terms of normal modes ${ }^{7}$

$$
p^{\prime}=(A \cos k z+B \sin k z) I_{m}(k r) e^{\beta t+i m \theta}
$$

Substituting eq 16 into eq 10 , we obtain

$$
\begin{aligned}
\xi_{\mathrm{r}} & =-(\mathrm{A} \cos \mathrm{kz}+\mathrm{B} \sin \mathrm{kz}) \frac{\mathrm{k}}{\rho \beta^{2}} \mathrm{I} \mathrm{m}^{\prime}(\mathrm{kr}) \mathrm{e}^{\beta \mathrm{t}+\mathrm{im} \theta} \\
\xi_{\theta} & =-(\mathrm{A} \cos \mathrm{kz}+\mathrm{B} \sin \mathrm{kz}) \frac{\mathrm{im}}{\mathrm{r} \rho \beta^{2}} \mathrm{I} \mathrm{m}(\mathrm{kr}) \mathrm{e}^{\beta \mathrm{t}+\mathrm{im} \theta} \\
\xi_{\mathrm{z}} & =(\mathrm{A} \sin \mathrm{kz}-\mathrm{B} \cos \mathrm{kz}) \frac{\mathrm{k}}{\rho \beta^{2}} \mathrm{I} \mathrm{m}(\mathrm{kr}) \mathrm{e}^{\beta \mathrm{t}+\mathrm{im} \theta}
\end{aligned}
$$

\section{Stationary Wave Conditions}

1. Boundary Conditions (eq 13) at $z=0, L$. Substituting eq 19 into eq 13, we obtain the stationary

(7) Chandrasekhar, S. Hydrodynamicand Hydromagnetic Stability; Oxford University Press: 1961.

(8) Courant, R.; Hilbert, D. Methods of Mathematical Physics; Wiley: New York, 1962; Vol. 2. 
waveconditions $B=0$ and $k=n \pi / L$ ( $n$ is a positiveinteger); then the solution of eqs 16-19 becomes

$$
\begin{gathered}
\mathrm{p}^{\prime}=\mathrm{A} \cos \frac{\mathrm{n} \pi \mathrm{Z}}{\mathrm{L}} \mathrm{I} \mathrm{m}\left(\frac{\mathrm{n} \pi \mathrm{r}}{\mathrm{L}}\right) \mathrm{e}^{\beta \mathrm{t}+\mathrm{im} \theta} \\
\xi_{\mathrm{r}}=-\frac{\mathrm{n} \pi \mathrm{A}}{\rho \beta^{2} \mathrm{~L}} \cos \frac{\mathrm{n} \pi \mathrm{z}}{\mathrm{L}} \mathrm{I}_{\mathrm{m}}\left(\frac{\mathrm{n} \pi \mathrm{r}}{\mathrm{L}}\right) e^{\beta \mathrm{t}+\mathrm{im} \theta} \\
\xi_{\theta}=-\frac{\mathrm{imA}}{\rho \beta^{2} \mathrm{r}} \cos \frac{\mathrm{n} \pi \mathrm{Z}}{\mathrm{L}} \mathrm{I}_{\mathrm{m}}\left(\frac{\mathrm{n} \pi \mathrm{r}}{\mathrm{L}}\right) e^{\beta \mathrm{t}+\mathrm{im} \theta} \\
\xi_{\mathrm{z}}=\frac{\mathrm{n} \pi \mathrm{A}}{\rho \beta^{2} \mathrm{~L}} \sin \frac{\mathrm{n} \pi \mathrm{z}}{\mathrm{L}} \mathrm{I}_{\mathrm{m}}\left(\frac{\mathrm{n} \pi \mathrm{r}}{\mathrm{L}}\right) e^{\beta \mathrm{t}+\mathrm{im} \theta}
\end{gathered}
$$

2. Behavior of the Axisymmetric Disturbance (m $=\mathbf{0}$ ). Applying the contact conditions of a soap film adhering to the edges of the two disks, we can prove that there only exist integer whole waves; i.e., $n \geq 2$ is any even number for axisymmetric disturbance $(m=0)$ of the cylindrical soap bubble.

In fact, due to the contact conditions of the soap film adhering to the edges of the two disks, the fluid volume enclosed by the film must be constant. For an axisymmetric disturbance $(m=0)$, the disturbing displacement $\zeta(a, z, t)$ of the film $(r=a)$ in a general form is

$$
\zeta(a, z, t)=\sum_{n=1}^{\infty} \zeta(a, n, t) \sin \frac{n \pi z}{L}
$$

All normal modes $\sin n \pi z / L$ areindependent of each other. Therefore, theconclusion needs only to be proven for every mode; i.e., take eq 24 as

$$
\zeta=\zeta(a, n, t) \sin \frac{n \pi z}{L}
$$

Applying the rule of volume conservation of fluid in a bubble and eq 25, we obtain

$$
\begin{aligned}
& 0=\iint_{\tau^{\prime}} \int d \tau-\pi a^{2} L=\int_{0}^{L} 2 \pi \xi d z= \\
& \left\{\begin{array}{ll}
0 & (n=2 q) \\
\frac{2 \pi a}{2 q-1} \zeta & (a, 2 q-1, t)
\end{array}(q=1,2,3, \ldots)\right.
\end{aligned}
$$

From thekinematics boundary condition (eq 7), wehave

$$
u_{r}=\frac{\partial \zeta}{\partial t}=\frac{\partial \xi_{r}}{\partial t}=\frac{\partial \xi_{r}(a, 2 q-1, t)}{\partial t}=0 \quad(r=a)
$$

for any time, which means the film did not have a disturbance of $m=0, n=2 q-1(q=1,2,3, \ldots)$.
Again from eqs $8-10$, we obtain

$$
p^{\prime}=-\sigma\left[\frac{\zeta}{a^{2}}+\frac{1}{a^{2}} \frac{\partial^{2} \zeta}{\partial \theta^{2}}+\frac{\partial^{2} \zeta}{\partial z^{2}}\right]=0 \quad(r=a)
$$

and

$$
\begin{gathered}
\frac{\partial \mathbf{p}^{\prime}}{\partial \mathrm{n}}=\frac{\partial \mathbf{p}^{\prime}}{\partial \mathrm{r}}=-\rho \frac{\partial^{2} \xi_{\mathrm{r}}}{\partial \mathrm{t}^{2}}=-\rho \frac{\partial \mathrm{u}_{\mathrm{r}}}{\partial \mathrm{t}}=0 \quad(\mathrm{r}=\mathrm{a}) \\
\frac{\partial \mathbf{p}^{\prime}}{\partial \mathrm{n}}=\frac{\partial \mathbf{p}^{\prime}}{\partial \mathrm{z}}=-\rho \frac{\partial^{2} \xi_{\mathrm{z}}}{\partial \mathrm{t}^{2}}=-\rho \frac{\partial \mathrm{u}_{\mathrm{z}}}{\partial \mathrm{t}}=0 \quad(\mathrm{z}=0, \mathrm{~L})
\end{gathered}
$$

On the basis of the maximum principle of a harmonic function (eq 8), the disturbing pressure $p^{\prime}$, as a harmonic function $\left(\nabla^{2} p^{\prime}=0\right)$ with the boundary conditions in eqs $28-30$, must be $p^{\prime} \equiv 0$. That means the wholefluid in the bubble is not disturbed. From here, we get a stationary wave condition $m=0$, where $n=2 q$ is an even number.

\section{Dispersion Relation and Stability Conditions}

Substituting the solutions of eqs $20-23$ into eq 12 , we get the dispersion relation as follows

$$
\beta^{2}=-\frac{\sigma}{\rho a^{3}}\left[m^{2}-1+\frac{n^{2} \pi^{2} a^{2}}{L^{2}}\right] \frac{n \pi a}{L} \frac{I_{m}{ }^{\prime}\left(\frac{n \pi z}{L}\right)}{I_{m}\left(\frac{n \pi z}{L}\right)}
$$

where $m=0, n=2 q$ for even numbers or $m \geq 1, n \geq 1$ for integers.

For a fluid column of length $L$, and replacing $n \pi a / L$ by ka, we get

$$
\beta^{2}=-\frac{\sigma}{\rho a^{3}}\left(m^{2}-1+k^{2} a^{2}\right) \frac{\mathrm{kal}_{m}{ }^{\prime}(\mathrm{ka})}{\mathrm{I}_{\mathrm{m}}(\mathrm{ka})}
$$

For an infinite fluid column, eq 32 is the same as eq 147 of ref 7, paragraph 111.

Since $\mathrm{xI}_{\mathrm{m}}{ }^{\prime}(\mathrm{x}) / \mathrm{I}_{\mathrm{m}}(\mathrm{x})$ is positive for all nonzero real $\mathrm{x}$, it fol lows from the dispersion relation (eq 31) that $\beta^{2}<0$ for all $n$ if $m \geq 1$ or $n=2 q>2$ if $m=0$. Under theseconditions $\beta$ is an imaginary number, and oscillations of fluid and film sustain themselves without growth or decay. The case $m \neq 0$ corresponds toa nonaxisymmetricdisturbance, and $\mathrm{m}=0$ corresponds to axisymmetric disturbances, so we may conclude that if the disturbances are nonaxisymmetric, thebubbleis al ways stable. On theother hand, $\beta^{2}>0$ for $\mathrm{n}=2$ if $\mathrm{m}=0$ indicates a uniquecritical unstable mode. If and only if $L>2 \pi a$, the bubble between the two disks with radius a is unstable, which is well consistent with the experimental results.

LA970595K 